\title{
The efficacy and mechanism of platelet-rich plasma on the myogenic differentiation process of adipose- derived stem cell: A bioinformatics and experimental study
}

\author{
Xiaochen Wang \\ Wuxi Ninth People's Hospital: Wuxi Hand Surgery Hospital \\ Wenyong Fei \\ Northern Jiangsu People's Hospital \\ Mingsheng Liu \\ Dalian Medical University
}

\section{Fanglin Shen}

Fudan University

Yu Liu ( $\square$ liuyuno9@163.com )

Wuxi Ninth People's Hospital: Wuxi Hand Surgery Hospital

sanjun Gu

Wuxi Ninth People's Hospital: Wuxi Hand Surgery Hospital

Changbao Wei

Wuxi Ninth People's Hospital: Wuxi Hand Surgery Hospital

\section{Qin Yin}

Wuxi Ninth People's Hospital: Wuxi Hand Surgery Hospital

Research article

Keywords: stem cells, PRP, growth, myogenic differentiation, bioinformatics analysis

Posted Date: February 5th, 2021

DOl: https://doi.org/10.21203/rs.3.rs-173133/v1

License: (9) This work is licensed under a Creative Commons Attribution 4.0 International License. Read Full License 


\section{Abstract}

Background: The adipose-derived stem cells (ADSCs) have been proved to be effective in the myogenic differentiation with the 5-Aza induction. However, the induction procedure definitly decreased the proliferation ability of ADSCs. The platelet-rich plasma (PRP), famous of its proliferating abilities, is widely used in the field of tissue regeneration. The purpose of this study is to determine the efficacy and mechanism of PRP on the myogenic differentiation process.

Method: The published gene expression profile, GSE 70918, which compared the efficacy of PRP and platelet-rich plasma (PPP) on the tendon fibroblast was obtained from the Gene Expression Omnibus (GEO) database.The further protein-protein interaction (PPI) network Gene Ontology (GO) and Kyoto Encyclopedia of Genes and Genomes (KEGG) analysis was performed based on differentially epressed genes(DEGs). Furethermore, the micro-RNA-mRNA interaction network was analyzed and visualized by cytoscape. Finally, the in vitro experimental cell research was performed to certify the efficay of PRP on myogenic differentiation.

Results: From our analysis, 148 DEGs, 87 up-regulated and 61 down-regulated, were obtained from the gene profile. The PPI network showed that the inflammatory factors as IL-1A, CXCL, CXCR etc. were significantly down-regulated and the muscle functional related genes as MYLC and MYO7A were significantly up-regulated. The GO and KEGG analysis demonstrated that up-regulated DEGs were enriched in focal adhesion, dilated cardiomyopathy, hypertrophic cardiomyopathy and calcium signaling pathway which were correlated to muscle function. Meanwhile, the microRNA-mRNA interaction network were estabilished based on DEGs. The in vitro experimental showed that PRP can significantly increase the proliferation ability of ADSCs during myogenic introduction and increase the expression of troponin and myosin which were correlate to the function of mature muscle.

Conclusions:The research showed that PRP is an effective way to enhance the proliferation ability during the myogenic differentiation and increase the expression of muscle functional related protein.

\section{Introduction}

The atrophy of the muscular tissue has been an issue perplexed orthopedist, aimed at repairing damaged tissues in surgery, for the postoperative complications it takes. For example, the rotator cuff tear, a chronic courses disease despaired the supraspinatus, owns a high re-tear rates of $94 \%$.[1]for the muscular atrophy as a main cause from Takashi Hashimoto, MD study. [2]As for the Duchenne Muscular Dystrophy, originated in the muscular atrophy, there still remains no effective method to hold back the proceeding of this disease[3]. Muscular tissue, originated from the mesoderm of our embryo, barely owns the regenerative ability for the terminal differentiating forms they are. Therefore, invent an effective method to regenerate the damaged muscular tissue is urgently needed.

The stem cells, which owns multidirectional differentiating abilities, has been promisingly expected to regenerate the damaged tissue and guide the clinical treatments. The Adipose-derived stem 
cells was firstly published in 2002 and they proved this type of stem cells own multilineage mesodermal differentiating potential which include the muscular tissue[4]. From the previous studies, the ADSCs can be induced into myocyte with various inductions as 5-Aza, boron, Cyclic uniaxial stress, etc. [5-7]And among them, the 5-Aza was set as a relative stabilized induction with specific myogenic differentiation ability not only in the ADSCs but also in the other MSCs. However, during the induction, there always exists the reduction of cell proliferation ability cause of the toxic effect of the inducers. The previous study showed that the cell viability was significantly reduced after 5-Aza induction. Therefore, an effective agent to prevent the toxic effect during induction is urgently needed.

Platelet-rich plasma, concentrated and collected from autologous platelet, is used in the field of stimulating tissue growth for the capacity of promote the proliferating ability. The PRP realize its effects mainly for the growth factors it contains which is critical to tissue regeneration, cellular recruitment and angiogenesis. In the previous research, PRP contains the ability of inducing the stem cells towards osteogenesis differentiation.[8] Meanwhile, PRP, combined with a TGF- $\beta$ inhibitor, turned to myogenic differentiation in Robi research.[9] As for the Michael J. McClure study, it reveals that PRP owns the ability of up-regulating the MRFs which represent the inchoate myogenic differentiation and down-regulate the MRFs which represent the terminal myogenic differentiation.[10] Whether PRP owns positive or negative effects on the myogenic differentiating process of ADSCs after the induction of 5-Aza is still under mystery.

\section{Materials And Methods}

\section{Microarray data obtained:}

Through the research from the GEO database, the gene expression profile, GSE 70918 (GPL 19271, Affymetrix Rat Gene 2.1 ST Array) was obtained. In this profile, there contained 4 samples, 2 of which were cultivated with PRP and the other 2 samples were cultivated with PPP. All of the 4 samples were included into our study.

\section{Identification of differently expressed genes (DEGs)}

The GEO2R tool was used to analysis the differentially expressed genes. And the threshold of the DEGs in our study was set as $|\log \mathrm{FC}|>1$ and P-value $<0.05$.

\section{(Protein-protein interaction) PPI network construction and module selection}

The PPI network based on commonly differentially expressed genes was construted by Search Tool for the Retrieval Interacting Genes (STRING) database. The Cytoscape(version 3.8.0) was used to analyze and visualized the correlations among genes. Molecular Complex Detection (MCODE; version 1.31) was used to determine the top 3 correlated gene modules.

\section{GO and KEGG pathway enrichment analysis construction}


The Database for Annotation, Visualization and Integrated Discovery (DAVID, version 6.8) was used to analyze the GO and KEGG functional enrichment based on the DEGs. Furthermore, the up-regulated and down-regulated DEGs were seperately analyzed from the online program.

\section{The miRNA-mRNA interaction analysis}

The differentially expressed miRNA was isolated from the profile. Online toll targetscan was used to determine the target mRNA of each miRNA. The venn tool was used to determine the commonly differentially expressed mRNA between the profile and target mRNA.

\section{Animal cares}

All animals received humane care in compliance with the Guidelines for the Care and Use of Laboratory Animals by the Department of Science and Technology of the People's Republic of China (2006). One 8week-old healthy New Zealand male, white rabbits (Provided by Jiangsu university experimental animal center of China) were used for this experiment.

\section{Rabbit Adiposederived Stem Cell Culture}

An eight-week-old New Zealand white male rabbit was prepared under general anesthesia, and a povidone-iodine solution was used to sterilize the hind leg before extracting the stem cells. The subpatellar fat pad was then harvested and collected in a $50 \mathrm{ml}$ centrifuge tube using a series of sterile surgical instruments. Several operations were carried out on a sterilized bench, as described below. The specimen was transferred to the culture dish where it was washed 3 times utilizing sterile phosphatebuffered saline (PBS). Superfluous tissues were wiped out, and the remaining tissues were minced finely

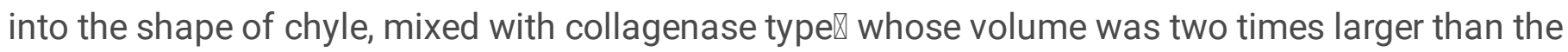
amount of tissue and moved to a $50 \mathrm{ml}$ centrifuge tube. The tube was next placed in constant temperature, and the table was set to vibration mode for 90 minutes to digest the tissue. At the end of digestion, the tissues were transferred into a centrifuge machine, and the rotator speed was set at 1700 r/min, which lasted 10 minutes. The top content of the centrifuged tube was blended with a $5 \mathrm{ml}$ pipette and centrifuged at $2000 \mathrm{r} / \mathrm{min}$ for 3 minutes. The top content and supernatant were discarded, leaving the lower white block mass content in the tube.

DMEM culture medium was added to the remaining tissue content left in the tube, and the mixture was centrifuged at $1500 \mathrm{r} / \mathrm{min}$ for 10 minutes, after which the supernatant was discarded, leaving just the lower part of the solution. This remaining tissue content was filtered with a $70 \mathrm{~nm}$ filter screen, leaving a liquid that was collected in a $15 \mathrm{ml}$ centrifuge tube. After centrifuging the liquid for 3 minutes at 800 $\mathrm{r} / \mathrm{min}$ and discarding the supernatant, the lower content containing the stem cells was obtained finally. Subsequently, we inoculated the stem cells into the flask filled with $5 \mathrm{ml}$ DMEM of fetal bovine serum and observed the morphological characteristics under the divert microscope. The cells were cultured in a Rsbiotech in a $5 \%$ CO2-saturated humidity pontoon at $37^{\circ} \mathrm{C}$, with the DMEM of fetal bovine serum subjected to culture and change every three days.

\section{Tetrazolium method DMTT}


The passage-3 ADSCs and the passage- 5 BMSCs were digested and diluted, and the mixture was transferred to a 96 -well culture plate at $100 \mu \mathrm{l}$ cell suspension per well and incubated at $37^{\circ} \mathrm{C}$, in a $5 \%$ CO2-saturated humidity float tank for 24 hours. 5-Aza was then added to each well containing ADSCs and the BMSCs at concentrations of $0,10,20,30,40 \mu \mathrm{mol} / \mathrm{I}$. The BMSCs groups were labeled groups A, $\mathrm{B}$, $C, D$, and $E$, and the ADSCs group were labeled groups F, G, H, I, and J. Following incubation under the same conditions as before for 24 hours, we added $50 \mu$ MTT solution to each well and incubated again under the same conditions for another 4 hours, terminating the reaction of each well by aspirating the culture medium. To each well was added $150 \mu \mathrm{L}$ DSO solution after terminating the 4-hours of incubation, with the plate placed on the table concentrator. Finally, the absorbance of each well was measured at $550 \mathrm{~nm}(\mathrm{OD})$ to detect the cell viability under the induction of different concentrations of 5Aza.

\section{Induction of differentiation and division into groups of BMSCs and ADSCs}

ADSCs were induced by 0,10 , and $20 \mu \mathrm{mol} / /$ of 5 -Aza in groups $A, B$, and $C$, while ADSCs were induced by $20 \mu \mathrm{mol} / \mathrm{I} 5-\mathrm{Aza}$ and $0,20,40 \mu \mathrm{mol} / \mathrm{I}$ PRP in groups $\mathrm{D}, \mathrm{E}$, and F, respectively. Group A served as the ADSCs control group, and group D played the role of 5-Aza control group. Groups $B$ and $C$ and groups $D$ and $E$ were ADSCs and PRP experimental groups, respectively. The morphologic characteristics of ADSCs in different groups were observed and recorded under an inverted microscope at regular intervals of 1,3 , 5,7 , and 9 days after induction of the stated concentrations of 5-Aza and PRP.

\section{Immunohistochemical staining and Semi-quantitative analysis}

First, we digested and diluted the cells from each group 9 days after induction; then we added $1 \mathrm{ml}$ of the solution to each well of the 12-well culture plate that had a cell slide placed in every well in advance. We next extracted the slides from each well after culturing at $37^{\circ} \mathrm{C}$ in a $5 \% \mathrm{CO} 2$-saturated humidity incubator for 24 hours. We washed the slides once with $300 \mu \mathrm{PBS}$ and did not remove the liquid for 5 minutes until the cells were saturated. Next, we added $150 \mu \mathrm{l}$ \% paraformaldehyde fixative to every slide and left them undisturbed for 30 minutes before adding $150 \mu \mathrm{l} 0.1 \%$ Triton $\mathrm{x}-100$ to each and left at room temperature for 3 minutes. We washed the slide 3 times with $300 \mu$ PBS, each washing process lasting 5 minutes. The ensuing step was to drop $200 \mu \mathrm{l}$ 5\% BSA blocking solution to each slide and incubate in a 5\% CO2 incubator for 1 hour. Each slide was incubated overnight at $4^{\circ} \mathrm{C}$ after being mixed with $150 \mu \mathrm{l}$ a-SMA (1:200) diluted with 1\% BSA. The slides were washed with $300 \mu \mathrm{l}$ PBS 5 times the following day, each washing process lasting 5 minutes. $150 \mu$ lof the secondary antibody (1:200) diluted with $1 \%$ BSA was then added to each slide after the slides were blocked at room temperature with $200 \mu$ 1 1\% BSA for 15

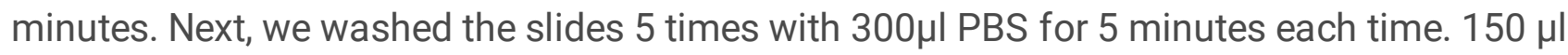
Hoechst33258 stain (C1011 Beyotine, China) was added to each slide in a dark environment and incubated for 30 minutes at room temperature. Finally, we observed the cells under a fluorescence microscope, photographed, and stored them.

A conventional laser scanning microscope can quantify fluorescently labeled tissues and display fluorescence changes in the direction of Z-axis. ImageJ software was used for photography and Prism 
Demo software for data statistics.

\section{RT-PCR}

Intracellular total RNA extraction: Pancreatic enzymes were used to digest the cells that were collected after centrifuging the liquid in the flask. Next, equal volumes of Trizol lysate were added to enable the collected cells to split and decompose. The schizolytic cells were then transferred into another tube without RNA enzymes, and $200 \mu \mathrm{l}$ pre-cooling chloroform was added per milliliter of Trizol, and the mixture was centrifuged for 15 minutes. The supernatant was absorbed after centrifugation and transferred into another tube without RNA enzymes, where an identical volume of isopropanol was added to the tube, followed by another centrifugation. This later centrifugation yielded RNA sediments that were preserved in a $-20^{\circ} \mathrm{C}$ surrounding for 30 minutes. The sediments were washed with $75 \%$ ethyl alcohol and centrifuged for 5 minutes, and the supernatant was discarded after washing and centrifuging the sediments twice.

cDNA synthesis: The reverse transcription system was prepared using a reverse transcription kit according to instructions provided in the protocol of the kit.

Polymerase chain reaction:

1. 1. Primer synthesis: The primers for reverse-transcription PCR were designed and synthesized using the Primer Premier 5.0 software. The internal reference was the housekeeping gene GAPDH.

2. 2. Real time-PCR reaction system: the SYBR Green PCR kit was used to carry out procedures in the real-time PCR reaction system.

3. 3. PCR amplification conditions: The real-time detector was used to detect the reaction, with the following temperature program was set: $94^{\circ} \mathrm{C}$ for 10 minutes and 40 cycles of $94^{\circ} \mathrm{C}$ for 20 seconds, $55^{\circ} \mathrm{C}$ for 20 seconds, and $72^{\circ} \mathrm{C}$ for 20 seconds.

\section{Statistical analysis}

Graphpad 8.0.2 and R 4.0.2 were used to perform statistical analysis. Expressed data were shown as mean $\pm S D$. Student's $t$ test was used to evaluate the statistical significance of different 3 groups. $P$ value less than 0.05 was considered as significant.

\section{Results}

\section{Identification of DEGs}

The DEGs were identified based on the threshold which was set as $|\log \mathrm{FC}|>1$ and P.value $<0.05$. From the analysis, 148 DEGs including 61 down-regulated and 87 up-regulated genes were indentified.

\section{Protein-protein interaction (PPI) network construction and sub-modules isolation}


148 notes and 412 edges were included in the network (Fig. 1A). Furthermore, based on the MCOD app, the top 3 modules were selected and shown in Fig. 1B, C, D. From the MCOD app, 23 top correlated genes were selected: CXCL16,CCL2,GNG3,NPY,MMP3,CCL20, C3, CXCR7,

IL1A,CXCL1,PF4,PRSS23,MMP10,MMP9,GAS6,MSLN,MMP13, CHRDL1,IL6, IL33, GNA14,CCL7,AGTR1A. Of which there were 6 up-regulated and 17 down-regulated genes. The down-regulated genes were almost correlated to inflammatory reaction and the up-regulated genes were correlated to the cell signal transduction.

\section{GO and pathway enrichment analysis from the DEGs}

GO analysis was processed to determine the functional distributions of DEGs from three aspects, molecular function (MF), biological processes (BP) and cell composition (CC). (Fig. 2) The up-regulated genes were enriched in aging, regulation of cardiac muscle cell contraction, cell-matrix adhesion, positive regulation of cytosolic calcium ion concentration, calcium ion transport in BP (Fig. 2D), extracellular matrix, cell surface, proteinaceous extracellular matrix, extracellular space in CC (Fig. 2E) and calcium ion binding, scavenger receptor activity, protein homodimerization activity, roundabout binding in MF.

(Fig. 2F) The down-regulated genes were enriched in positive regulation of ERK1 and ERK2 cascade, response to lipopolysaccharide, cellular response to interleukin-1, inflammatory response in BP (Fig. 2G), extracellular space, extracellular region in $\mathrm{CC}$ (Fig. 2H) and chemokine activity, hormone activity, CCR2 chemokine receptor binding in MF (Fig. 2I).

The KEGG analysis results showed that the DEGs were enriched in Chemokine signaling pathway, Cytokine-cytokine receptor interaction, TNF signaling pathway. (Fig. 3A) Meanwhile, the up-regulated DEGs were enriched in Focal adhesion, Dilated cardiomyopathy, ECM-receptor interaction, PI3K-Akt signaling pathway, Hypertrophic cardiomyopathy (HCM), cGMP-PKG signaling pathway, Platelet activation.(Fig. 3B) The down-regulated DEGs were enriched in TNF signaling pathway, Chemokine signaling pathway, Cytokine-cytokine receptor interaction, Rheumatoid arthritis.(Fig. 3C)

According to the $\mathrm{GO}$ and KEGG analysis, the up-regulated genes were enriched in the cellular energetics and muscle functions, especially the activity of the calium in cells. What's more, the down-regulated genes were enriched in the inflammatory response. Therefore, we indicated that PRP can increas the cell viability during induction and can also increase the expression the calium related protein as troponin and myosin.

\section{MiRNA-mRNA interaction results}

From the DEGs, there were two miDEGs, miR-24-1 and mir3554 isolated. Mir-24-1 were correlated to 16 mDEGs (Figure 4A) and mir3554 (Figure 4B) were correlated to 12 mDEGs. The correlation network were shown in Figure 4C.

\section{The effects of PRP on the myogenic trend of ADSCs}

\section{Cell viability evaluated by MTT test}


The cells were set in 6 groups and each group was induced by 5-Aza with the same concentration, $20 \mu \mathrm{mol} / \mathrm{L}$. Apart from the 5-Aza, the different concentration of PRP, $0 \mu \mathrm{l} / \mathrm{ml}, 10 \mu \mathrm{l} / \mathrm{ml}, 20 \mu \mathrm{l} / \mathrm{ml}, 30 \mu \mathrm{l} / \mathrm{ml}$, $40 \mu \mathrm{l} / \mathrm{ml}, 50 \mu \mathrm{l} / \mathrm{ml}$, were added into the 6 different groups respectively. The MTT results were converted into figure in figure5B. The PRP, famous of the proliferating ability, do increase the cell viability whatever the concentration in the groups is. The cell viability was significantly improved by PRP. From the calculation, the IC50 of PRP was $19.4 \mu \mathrm{mol} / \mathrm{l}$.

\section{Morphology comparisons among different concentrations of PRP}

The morphology of the cells was observed, recorded and merged in fig5A for the final comparison. The group D, induced by $20 \mu \mathrm{mol} / \mathrm{I} 5-\mathrm{Aza}$ only, was set as the control group whose appearance has no significant change until the third day of induction. The cells were live in colonies and there were many died cells floating in the medium every time we changed the medium. In the experimental groups $E$ and $F$, the cells became into huger size and changed into the shape of oval at the first day. The cells live into bundles at first day and as time pass by, the died cells were less than group $D$ when compared at the same time point.

\section{The comparisons about the expression of actin by immunohistochemical stain}

Same to the previous experiments, the expression of actin was detected and showed in the fig5C. The hochest line manifest the living nucleus in different groups. Compared to group $D$, the group $E$ and $F$ own higher density of the nucleus. The a-SMA line showed the expression of actin in different groups. There showed no significant difference among the three groups in the volume of actin. The merge line manifested the differentiation ratio of each group and the ratios were calculated and presented at fig $5 \mathrm{~d}$. As the figure showed, the differentiating rate of group $D, E$ and $F$ were $0.117,0.123$ and 0.128 respectively. There was no apparent increase of the data no matter whatever the concentration of PRP was. It can be apparently obvious that the PRP has no effects on the synthesis of actin, a myogenic differentiation index, after the induction of 5-Aza.

\section{RT-PCR to compare the expression of myogenic gene, troponin and myosin}

In the stated three groups, the expression of the myogenic gene, troponin and myosin, corelating to the caluim activity, were detected by RT-PCR technology. The results were shown in the figure5e. The group D, induced by $20 \mu \mathrm{mol} / / 5$-Aza only, showed a basic volume of the expression in the myogenic process. In the comparisons of troponin, there showed a significant up-regulation after PRP clutivation. The expression of troponin was 1.002, 1.544 and .1.631 in group D, E and F respectively(Figure 5e). As for the myosin, significant increase of the expression in the experimental groups were displayed when compared to control group. The expression of myosin was 1.066, 1.412 and 1.517 in group D, E and $F$ respectively.(Figure 5e)

Between the two experimental groups, cultured with different concentrations of PRP, the expression of troponin and myosin showed little difference. 


\section{Discussion}

Stem cells, greatly promised to repair and regenerate damaged tissue, are notably famous of their potency of multi-directional differentiation. Meanwhile, the ADSCs, extracted from the autologous adipose tissue, were treated as a novel therapy to repair and regenerative the damaged tissue from the pharmacologic research to clinical research. [11-13]From the previous research, it can be apparently certified the ADSCs, originate from the mesoblast, own the ability of differentiating into bone, cartilage and muscular tissue whose origination are the same to the ADSCs.[14] Furthermore, the differentiating abilities of ADSCs have expanded to ectodermal and endodermal lineages.[4] Based on the differentiating abilities, the ADSCs were expected to play a vital role in the department of orthopedics.

With the rapid development of orthopedics technology, a great deal of diseases has been greatly handled and the postoperative recovery have been carefully implied. Both of the aspects led to a better result to the orthopedics patients. However, there still remained a number of diseases accompanied with the atrophy of muscular tissue as the Duchenne Muscular Dystrophy, the degenerated rotator cuff tear, the spinal muscular atrophy that lead to the poor outcomes after thorough therapy. [15-17]As the rotator cuff tear for an example, with the atrophy of supraspinatus, the postoperative re-tear rate increased apparently. Therefore, research a feasible method to prevent the atrophy process of local muscular tissue and promote the proliferation of the damaged muscular tissue is urgently needed in our clinical treatment.

PRP, concentrated autologous plasma, own rich volume of growth factors that not only promote the proliferation abilities of local tissue, but also promote the differentiation abilities of stem cells. There exists a great deal of cytokines that derive the stem cells into different terminal types. The factors TGF- $\beta$, VEGF, IGF- 1 and SDF- 1 showed acceleration in the myogenic procession and the factors $\beta$-FGF,TNF- $a$ showed inhibition in the myogenic procession. $[18,17]$ Although the specific effects of each factors have been researched, the combined effects on the myogenic process have barely researched. From the previous study, the PRP can not only induce the cells towards skeleton[8], but also induce the cells into muscular tissue when combined with TGF- $\beta$ inhibitor. [19]Seldom research has explored the myogenic differentiating ability of ADSCs when the cells were co-cultured by 5-Aza and PRP.

Our experiment examined the effects of PRP on the ADSCs myogenic process with the induction of 5-Aza. The results from the morphologic changes showed that the PRP demonstrated apparently proliferating abilities for the increasing density of cells on the morphologic figures and increasing the density of nucleus on the immunohistochemical staining figures. Meanwhile, the calium activity related genes as troponin and myosin were also up-regulated after PRP cultivation.

\section{Conclusion}

The ADSCs, extracted from the sub-patellar fat pad, own the myogenic differentiating ability with the induction of 5-Aza.The different concentrations of PRP were co-cultured with $20 \mu \mathrm{mol} / \mathrm{I}$ 5-Aza for 9 days 
and the results from morphologic changes to immunohistochemical staining and RT-PCR technology showed that PRP can increse the cell proliferating ability and the expression of troponin and myosin.

\section{Declarations}

\section{Ethics approval and consent to participate}

This study was approved by ethics of committee of Northern Jiangsu People's Hospital.

\section{Consent for publish}

Not applicable.

\section{Availability of data and materials}

The data used and analyzed during the current study are available from the corresponding author on reasonable request.

\section{Competing Interest}

The authors declare that they have no competing interests.

\section{Funding:}

This work was supported by the funds from the young medical key talent project of Jiangsu province (contract number QNRC2016458) and Medical and Public Health Technology Innovation and Application Project of Wuxi Science and Technology Bureau (contract number N202041)

\section{Authors' contributions}

Xiaochen Wang,Wenyong Fei and Yu Liu: conception and design, financial support, experiment, manuscript writing, final approval of manuscript.

Correspondence: Yu Liu

All authors read and approved the final manuscript.

\section{References}

1. Galatz LM, Ball CM, Teefey SA, Middleton WD, Yamaguchi K. The outcome and repair integrity of completely arthroscopically repaired large and massive rotator cuff tears. The Journal of bone joint surgery American volume. 2004;86(2):219-24. doi:10.2106/00004623-200402000-00002.

2. Hashimoto T, Nobuhara K, Hamada T. Pathologic evidence of degeneration as a primary cause of rotator cuff tear. Clin Orthop Relat Res. 2003(415):111-20.

doi:10.1097/01.blo.0000092974.12414.22. 
3. Hoffman EP, Brown RH Jr, Kunkel LM. Dystrophin: the protein product of the Duchenne muscular dystrophy locus. Cell. 1987;51(6):919-28. doi:10.1016/0092-8674(87)90579-4.

4. Zuk PA. The adipose-derived stem cell: looking back and looking ahead. Molecular biology of the cell. 2010;21(11):1783-7. doi:10.1091/mbc.E09-07-0589.

5. Mizuno H, Zuk PA, Zhu M, Lorenz HP, Benhaim P, Hedrick MH. Myogenic differentiation by human processed lipoaspirate cells. Plastic reconstructive surgery. 2002;109(1):199-209. doi:10.1097/00006534-200201000-00030. discussion $10-1$.

6. Apdik H, Dogan A, Demirci S, Aydin S, Sahin F. Dose-dependent Effect of Boric Acid on Myogenic Differentiation of Human Adipose-derived Stem Cells (hADSCs). Biol Trace Elem Res. 2015;165(2):123-30. doi:10.1007/s12011-015-0253-3.

7. Bayati V, Sadeghi Y, Shokrgozar MA, Haghighipour N, Azadmanesh K, Amanzadeh A, et al. The evaluation of cyclic uniaxial strain on myogenic differentiation of adipose-derived stem cells. Tissue Cell. 2011;43(6):359-66. doi:10.1016/j.tice.2011.07.004.

8. Fennis JP, Stoelinga PJ, Jansen JA. Mandibular reconstruction: a histological and histomorphometric study on the use of autogenous scaffolds, particulate cortico-cancellous bone grafts and platelet rich plasma in goats. Int J Oral Maxillofac Surg. 2004;33(1):48-55. doi:10.1054/ijom.2003.0452.

9. Kelc R, Trapecar M, Gradisnik L, Rupnik MS, Vogrin M. Platelet-rich plasma, especially when combined with a TGF-beta inhibitor promotes proliferation, viability and myogenic differentiation of myoblasts in vitro. PloS one. 2015;10(2):e0117302. doi:10.1371/journal.pone.0117302.

10. McClure MJ, Garg K, Simpson DG, Ryan JJ, Sell SA, Bowlin GL, et al. The influence of platelet-rich plasma on myogenic differentiation. J Tissue Eng Regen Med. 2016;10(4):E239-49. doi:10.1002/term.1755.

11. Nakagami H, Morishita R, Maeda K, Kikuchi Y, Ogihara T, Kaneda Y. Adipose tissue-derived stromal cells as a novel option for regenerative cell therapy. J Atheroscler Thromb. 2006;13(2):77-81. doi:10.5551/jat.13.77.

12. Nakagami H, Maeda K, Morishita R, Iguchi S, Nishikawa T, Takami Y, et al. Novel autologous cell therapy in ischemic limb disease through growth factor secretion by cultured adipose tissue-derived stromal cells. Arteriosclerosis, thrombosis, and vascular biology. 2005;25(12):2542-7. doi:10.1161/01.ATV.0000190701.92007.6d.

13. Sterodimas A, de Faria J, Nicaretta B, Pitanguy I. Tissue engineering with adipose-derived stem cells (ADSCs): current and future applications. Journal of plastic reconstructive aesthetic surgery: JPRAS. 2010;63(11):1886-92. doi:10.1016/j.bjps.2009.10.028.

14. Gang EJ, Jeong JA, Hong SH, Hwang SH, Kim SW, Yang IH, et al. Skeletal myogenic differentiation of mesenchymal stem cells isolated from human umbilical cord blood. Stem Cells. 2004;22(4):617-24. doi:10.1634/stemcells.22-4-617.

15. Davies MR, Garcia S, Tamaki S, Liu X, Lee S, Jose A, et al. Muscle stem cell activation in a mouse model of rotator cuff injury. Journal of orthopaedic research: official publication of the Orthopaedic Research Society. 2018;36(5):1370-6. doi:10.1002/jor.23679. 
16. Kolb SJ, Kissel JT. Spinal Muscular Atrophy. Neurologic clinics. 2015;33(4):831-46. doi:10.1016/j.ncl.2015.07.004.

17. Duan $\mathrm{C}$, Ren $\mathrm{H}$, Gao S. Insulin-like growth factors (IGFs), IGF receptors, and IGF-binding proteins: roles in skeletal muscle growth and differentiation. Gen Comp Endocrinol. 2010;167(3):344-51. doi:10.1016/j.ygcen.2010.04.009.

18. El-Sharkawy H, Kantarci A, Deady J, Hasturk H, Liu H, Alshahat M, et al. Platelet-rich plasma: growth factors and pro- and anti-inflammatory properties. Journal of periodontology. 2007;78(4):661-9. doi:10.1902/jop.2007.060302.

19. Alsousou J, Thompson M, Hulley P, Noble A, Willett K. The biology of platelet-rich plasma and its application in trauma and orthopaedic surgery: a review of the literature. The Journal of bone joint surgery British volume. 2009;91(8):987-96. doi:10.1302/0301-620x.91b8.22546.

\section{Figures}




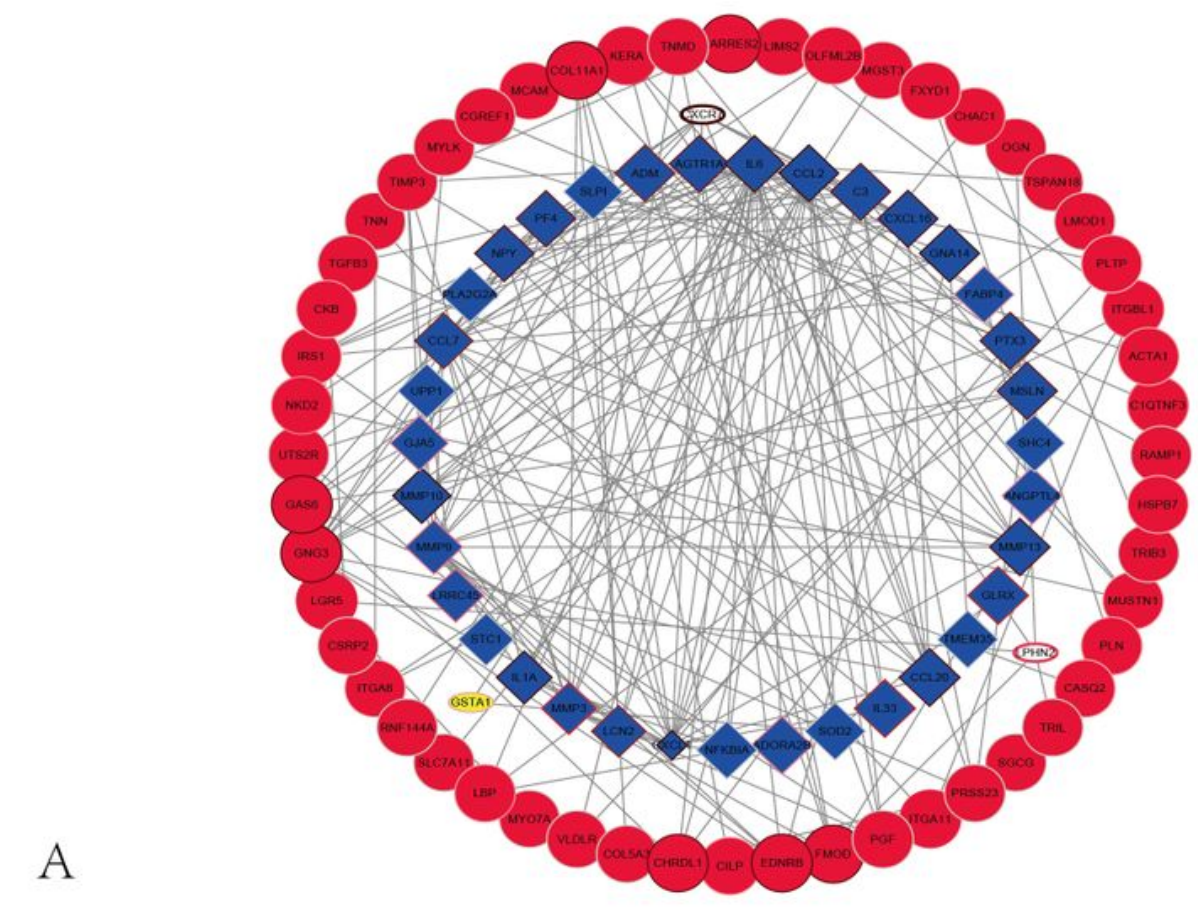

B

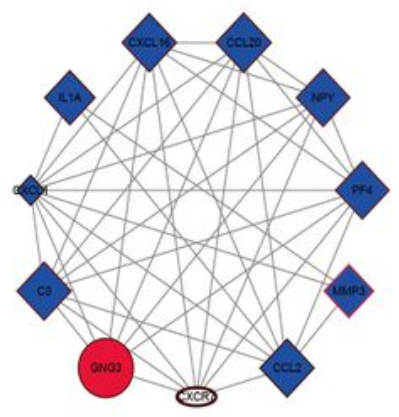

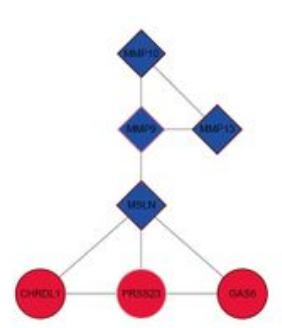

C

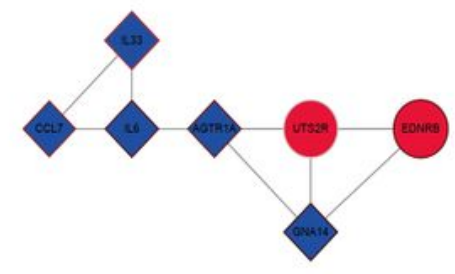

$\mathrm{D}$

\section{Figure 1}

A. 148 notes and 412 edges were included in the network. B. The top 3 modules were selected 


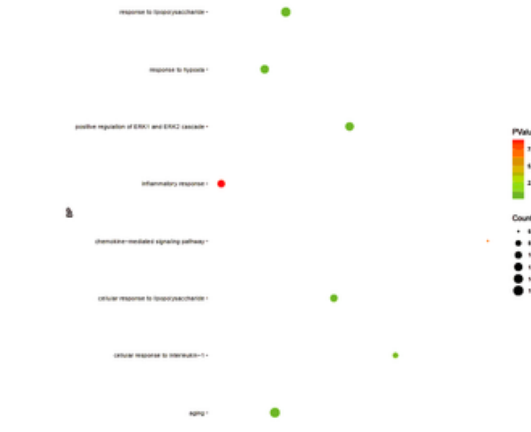

A

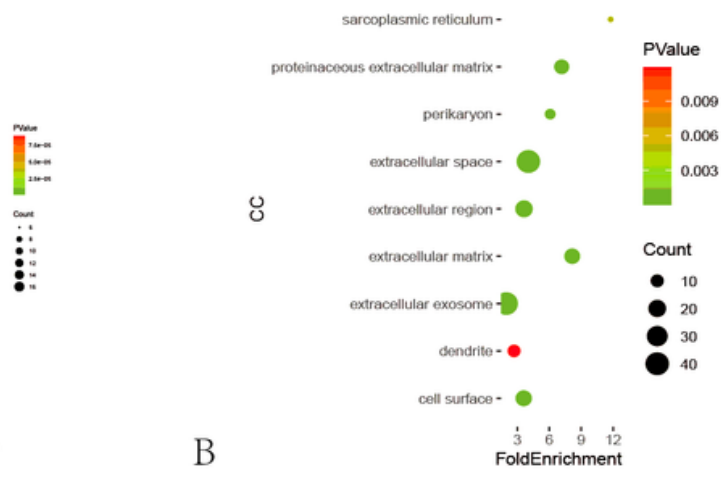

D

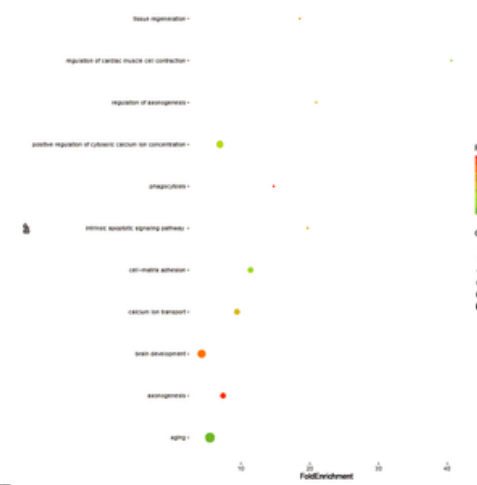

D

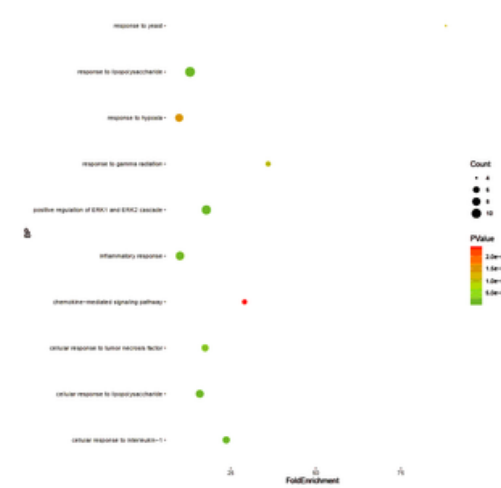

G

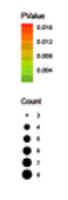

E
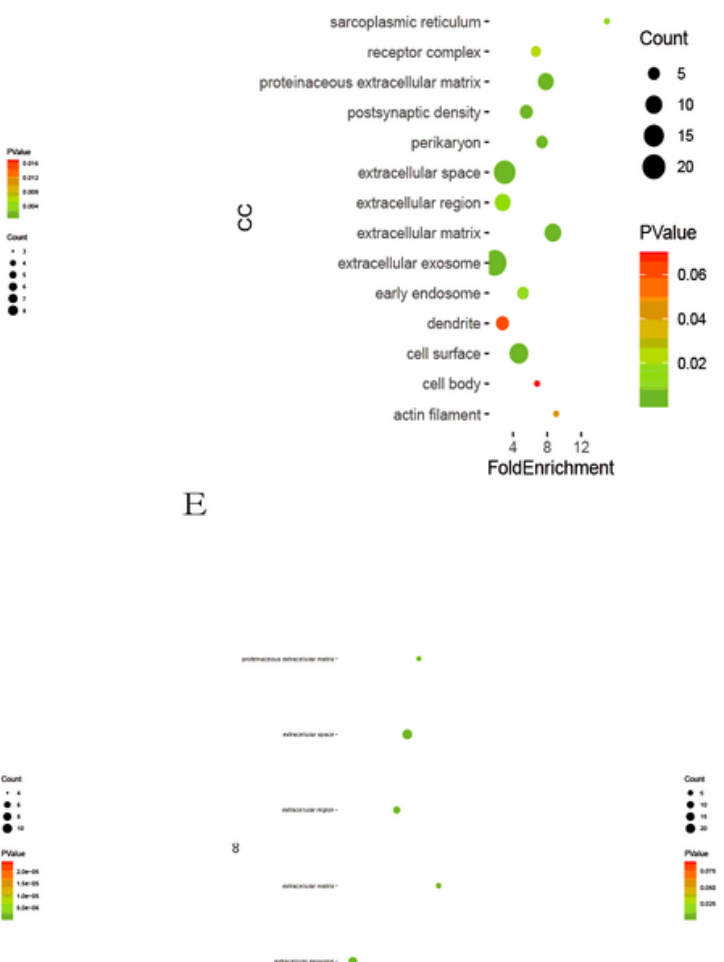

$\mathrm{H}$
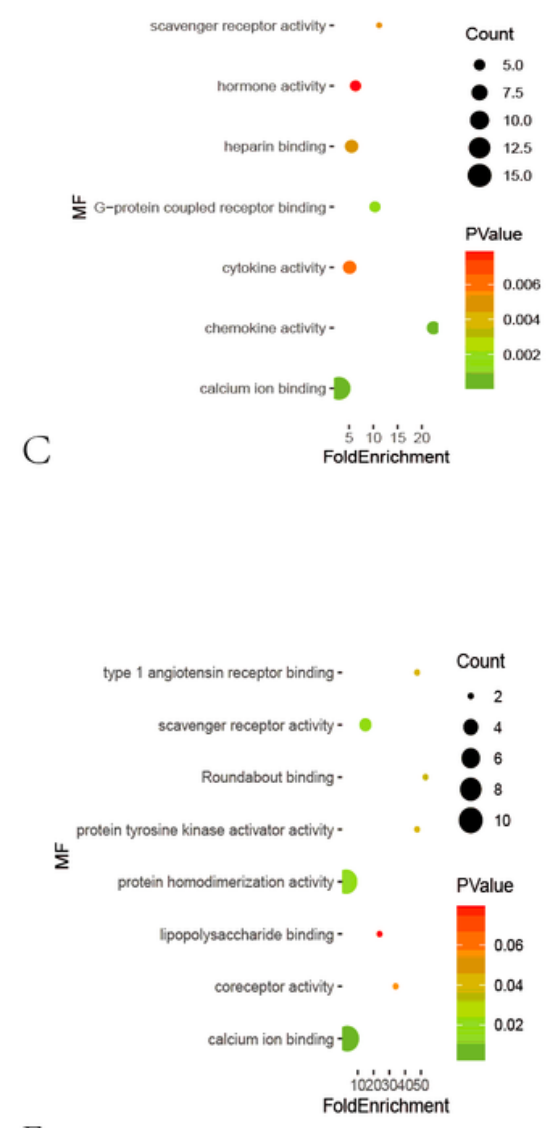

F

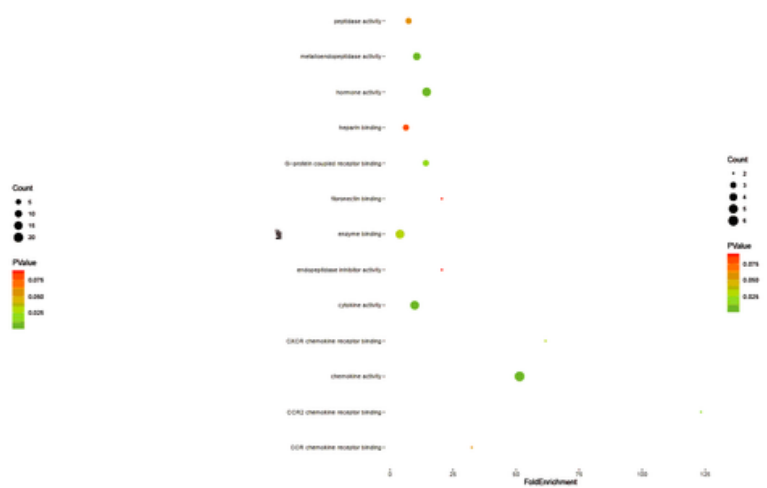

I

Figure 2

The up-regulated genes were enriched in aging, regulation of cardiac muscle cell contraction, cell-matrix adhesion, positive regulation of cytosolic calcium ion concentration, calcium ion transport in BP 

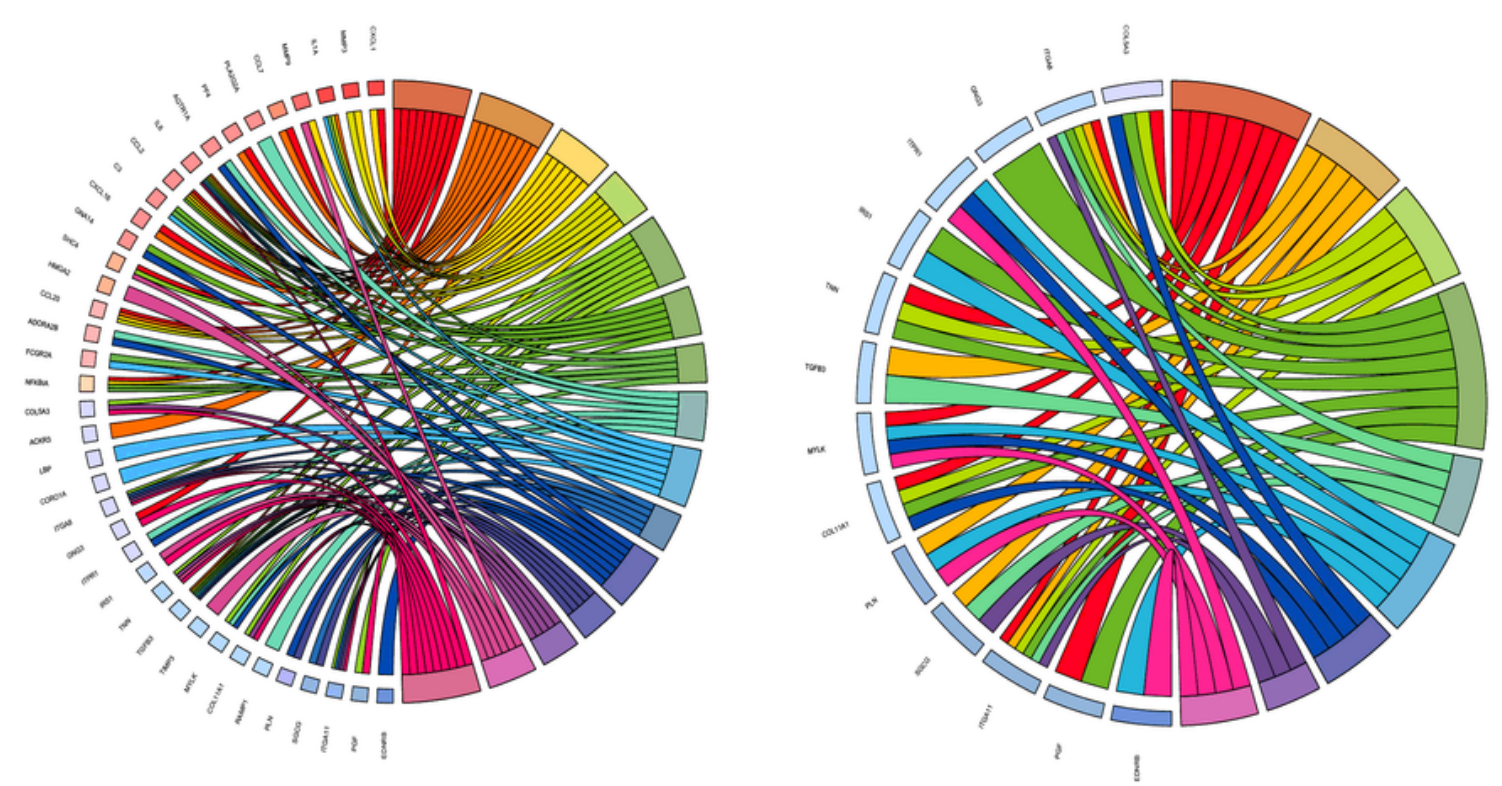

A
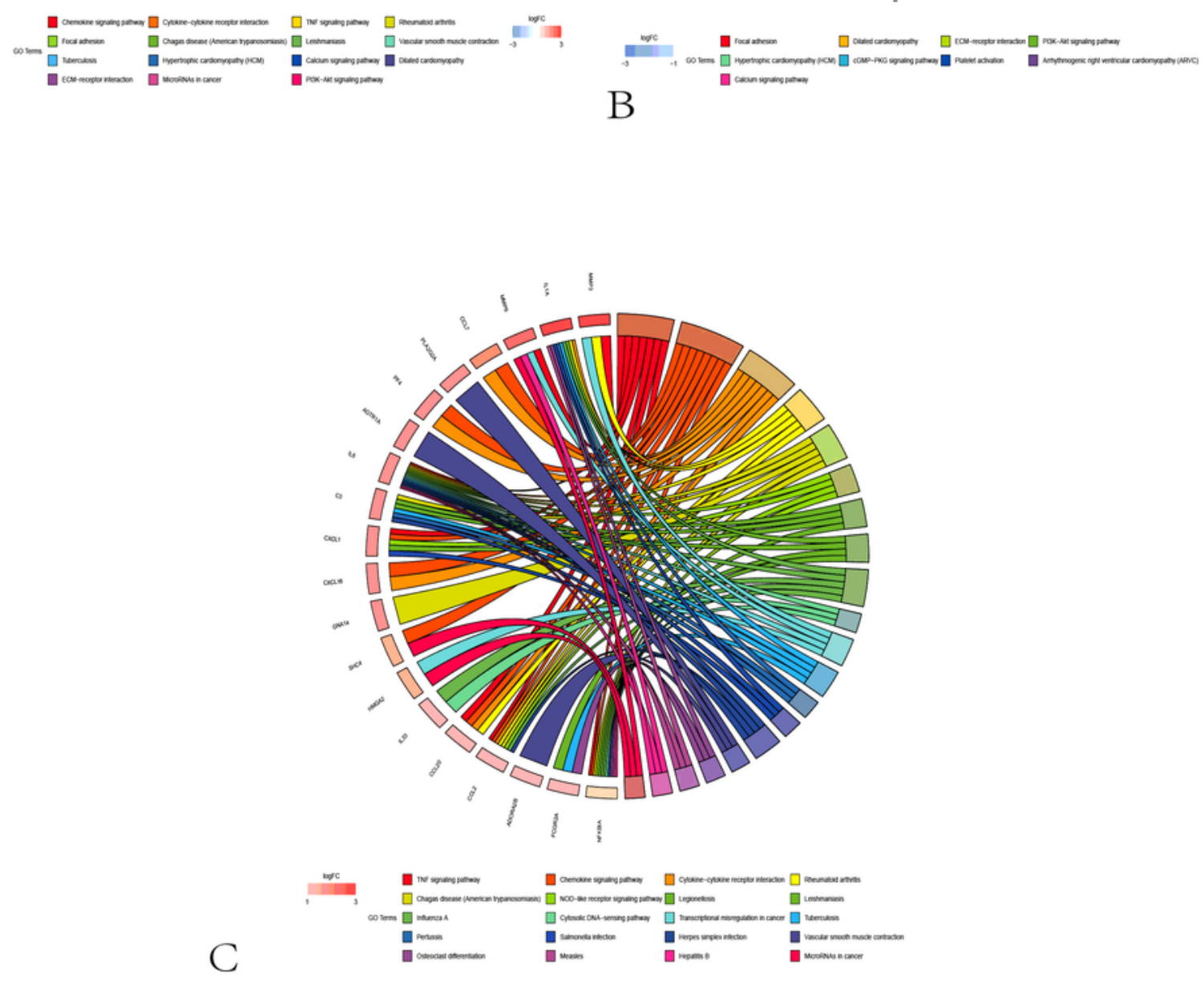

Figure 3

The KEGG analysis results showed that the DEGs were enriched in Chemokine signaling pathway, Cytokine-cytokine receptor interaction, TNF signaling pathway 


\begin{tabular}{|c|c|c|c|c|c|}
\hline RNA & $\log F C$ & $\mathrm{t}$ & $\mathrm{B}$ & P.Value & adj.P.Val \\
\hline MIR24-1 & -1.16 & $1.04 E+01$ & 1.18021 & 0.00027834 & 0.1201 \\
\hline $\mathrm{SHC} 4$ & 1.53 & $2.34 \mathrm{E}+01$ & 0.00000803 & 0.0597 & 3.26093 \\
\hline SOD2 & 1.21 & $1.59 E+01$ & 0.00004398 & 0.0805 & 2.47102 \\
\hline CYP7B1 & 1.14 & $-1.51 E+01$ & 0.00005489 & 0.084 & 2.33999 \\
\hline NABP1 & 1.13 & $-1.47 E+01$ & 0.00006181 & 0.0857 & 2.26707 \\
\hline VLDLR & -1.05 & $1.41 E+01$ & 0.00007383 & 0.0868 & 2.15427 \\
\hline SULF2 & -1.12 & $-1.28 \mathrm{E}+01$ & 0.00011314 & 0.0965 & 1.86593 \\
\hline IRS1 & -1.18 & $-1.14 \mathrm{E}+01$ & 0.0001888 & 0.1051 & 1.48837 \\
\hline ADARB1 & -1.29 & $1.12 \mathrm{E}+01$ & 0.0002047 & 0.1081 & 1.42569 \\
\hline OGN & -1.46 & $1.06 \mathrm{E}+01$ & 0.00025819 & 0.1199 & 1.24128 \\
\hline SBSPON & -1.48 & -8.97 & 0.00052138 & 0.1322 & 0.6449 \\
\hline ITGBL1 & -1.54 & 8.93 & 0.00053138 & 0.1326 & 0.62802 \\
\hline PARM1 & -1.82 & -8.81 & 0.00056437 & 0.1363 & 0.57429 \\
\hline PRSS23 & -1.96 & -8.09 & 0.0008058 & 0.1607 & 0.24929 \\
\hline ITGA11 & -1.96 & -6.97 & 0.00149838 & 0.2013 & -0.34403 \\
\hline FMOD & -2.43 & -6.29 & 0.00228841 & 0.2299 & -0.76616 \\
\hline CILP & -2.83 & -4.56 & 0.00807912 & 0.3148 & -2.08328 \\
\hline RNA & $\log F C$ & $\mathrm{t}$ & B & P.Value & adj.P.Val \\
\hline MIR3554 & 1.01 & -8.46 & 0.41879 & 0.00067034 & 0.1535 \\
\hline MMP13 & 1.46 & $-1.02 E+01$ & 0.00030455 & 0.1218 & 1.10618 \\
\hline NABP1 & 1.13 & $1.06 \mathrm{E}+01$ & 0.00025819 & 0.1199 & 1.24128 \\
\hline ADAM23 & -1.01 & 4.42 & 0.00906187 & 0.3243 & -2.20629 \\
\hline VLDLR & -1.05 & $2.34 \mathrm{E}+01$ & 0.00000803 & 0.0597 & 3.26093 \\
\hline RNF144A & -1.07 & $-1.37 E+01$ & 0.00008503 & 0.0868 & 2.06158 \\
\hline LGR5 & -1.09 & -9.18 & 0.00047321 & 0.1321 & 0.7304 \\
\hline EPHA4 & -1.17 & -7.89 & 0.00089666 & 0.1653 & 0.14945 \\
\hline FAT3 & -1.24 & -8.03 & 0.00083178 & 0.1614 & 0.21975 \\
\hline PLXDC2 & -1.32 & $-1.23 E+01$ & 0.00013297 & 0.0996 & 1.7505 \\
\hline COL11A1 & -1.35 & 6.36 & 0.00218295 & 0.227 & -0.71853 \\
\hline C1QTNF3 & -1.62 & 5.35 & 0.00435522 & 0.2752 & -1.42885 \\
\hline EDNRB & -2.72 & 7.76 & 0.00096361 & 0.1667 & 0.08158 \\
\hline
\end{tabular}

C

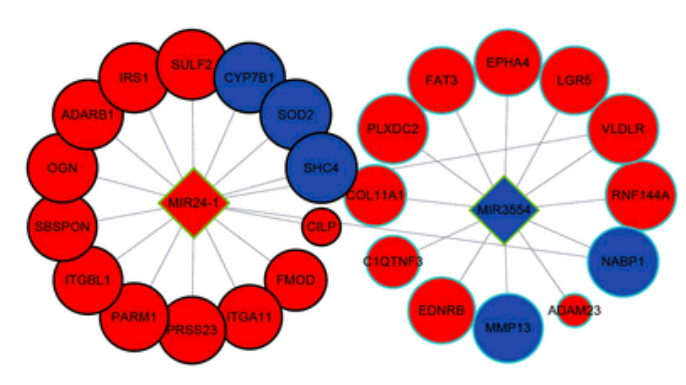

\section{Figure 4}

From the DEGs, there were two miDEGs, miR-24-1 and mir3554 isolated. Mir-24-1 were correlated to 16 mDEGs (Figure 4A) and mir3554 (Figure 4B) were correlated to 12 mDEGs. The correlation network were shown in Figure 4C. 

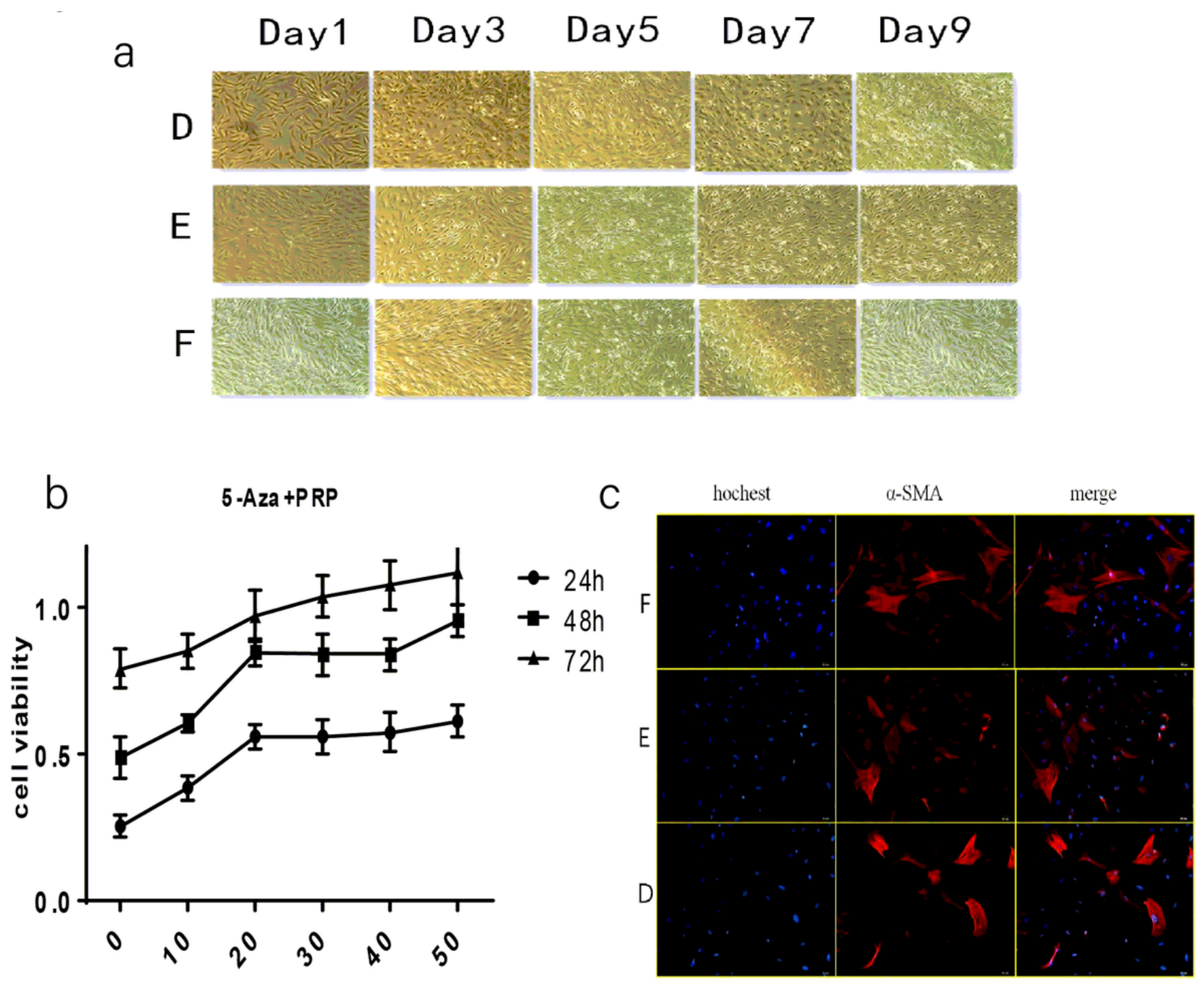

Figure 5

The MTT results 